\title{
National Committee for Quality Assurance (NCQA) Patient-Centered Medical Home (PCMH) Recognition Is Suboptimal Even Among Innovative Primary Care Practices
}

\author{
Karissa A. Hahn, MPH, Martha M. Gonzalez, BA, Rebecca S. Etz, PhD, \\ and Benjamin F. Crabtree, $P h D$
}

The National Committee for Quality Assurance (NCQA) has promoted patient-centered medical home (PCMH) recognition among primary care practices since 2008 as a standard indicator of which practices have transformed into medical homes. A 40\% PCMH adoption rate among a large national cohort of identified practices with innovative staffing $(n=131)$ calls into question whether the NCQA recognition process is truly transformative and patient-centered or simply another certificate to hang on the wall. (J Am Board Fam Med 2014;27:312-313.)

Keywords: Medical Home, Patient-Centered Care, Practice-based Research

The NCQA first introduced PCMH recognition in 2008. The goal of this recognition is to improve the quality of care. ${ }^{1}$ Previous research has shown that the decision to pursue NCQA PCMH recognition often is based on financial incentives. ${ }^{2,3}$ In addition, the desire to become a patient-centered practice and to seek NCQA recognition are not necessarily aligned: "PCMH recognition per se at times amounts to a small incentive to change labels." 2

\footnotetext{
This article was externally peer reviewed.

Submitted 4 October 2013; revised 27 January 2014; accepted 29 January 2014.

From the Department of Family Medicine and Community Health, Rutgers University, Robert Wood Johnson Medical School, New Brunswick, NJ (KAH, BFC); and the Department of Family Medicine and Population Health, Virginia Commonwealth University, Richmond (MMG, RSE).

Funding: none.

Conflict of interest: none.

Disclaimer: Policy briefs represent the opinions of the authors and not necessarily those of the American Board of Family Medicine.

Corresponding author: Karissa A. Hahn, MPH, Rutgers University, Robert Wood Johnson Medical School, 1 Worlds Fair Drive, Somerset, NJ 08873 (E-mail: karissahahn@ alumni.rutgers.edu).
}

See Related Commentary on Page 309.
True transformation to a $\mathrm{PCMH}$ requires a dramatic shift in practice culture and mental models. ${ }^{4}$

As part of a study of primary care workforce innovations funded by The Robert Wood Johnson Foundation, we consulted with 387 national experts in primary care research, using both purposeful and snowball sampling, to identify a set of 131 practices that experts knew to be (1) high quality, (2) clinically excellent settings, and (3) doing something innovative with their staffing (Table 1). We conducted 1-hour phone interviews with these innovative primary care practices. ${ }^{5}$ Among other topics, we asked practices whether they were recognized as PCMHs by the NCQA and, if not, whether they were pursuing recognition.

While the PCMH recognition level among all primary care practices at the time of this study was approximately $5 \%,{ }^{1,6}$ we were surprised to find that among our sample of innovative practices who had already made substantial transformations, NCQA PCMH recognition levels rose only to $40 \%$ (Figure 1). Several key themes emerged from practice members' comments. Many practices questioned whether PCMH recognition was a meaningful credential (34\%), often citing cost of recognition and lack of payer incentives. Among the $15 \%$ of practices not seeking recognition, $28 \%$ said they were already us- 
Table 1. Characteristics of Innovative Practices

\begin{tabular}{lc}
\hline Characteristics & Sites, n (\%) \\
\hline Size & \\
Large ( $\geq 10$ PCPs) & $45(34)$ \\
Medium (5-9 PCPs) & $48(37)$ \\
Small (2-4 PCPs) & $31(24)$ \\
Solo (1 PCP) & $7(5)$ \\
Geographic area & \\
Urban & $53(40)$ \\
Suburban & $38(29)$ \\
Rural & $35(27)$ \\
Frontier & $3(2)$ \\
Mix (urban/suburban) & $2(2)$ \\
Organization & \\
Private & $68(52)$ \\
FQHC/CHC & $47(36)$ \\
Academic & $9(7)$ \\
Hospital owned & $7(5)$ \\
Clinical focus & \\
FM & $67(51)$ \\
Multiple & $32(24)$ \\
FM and IM & $12(9)$ \\
IM & $9(7)$ \\
Pediatrics & $7(5)$ \\
Pediatrics/FM, pediatrics/IM & $4(3)$ \\
\hline
\end{tabular}

CHC, community health center; FHQC, federally qualified health center; FM, family medicine; IM, internal medicine; $\mathrm{PCP}$, primary care physician.

ing the PCMH model, whereas others felt that the recognition process was unnecessary or continued to be a moving target (5\%). Even among practices that were recognized, some felt it did little to promote meaningful improvements in patient experience or health outcomes (9\%). Practices also reported that a focus on other improvements in quality of care was at times a hindrance to the recognition process.

The decision to pursue $\mathrm{PCMH}$ recognition among the innovative practices involved in this study was, in many cases, based on financial incentives and not necessarily on a belief that the recognition would result in higher quality of care. Suboptimal levels of NCQA PCMH recognition among these practices calls into question whether the process of NCQA recognition is one that is truly transformative for primary care practices, resulting in high-quality, patient-centered care, or simply a label. Adapting the recognition criteria to include measures that are meaningful to practices as well as pushing insurers to provide incentives to
Figure 1. Patient-centered medical home (PCMH) recognition among innovative primary care practices, June 2012. While we focus on recognition by the National Committee of Quality Assurance (NCQA), other entities such as the Veterans Administration, state governments, and insurance companies also have PCMH recognition programs. In our data set, fewer than 10 practices were certified through one of these other entities.

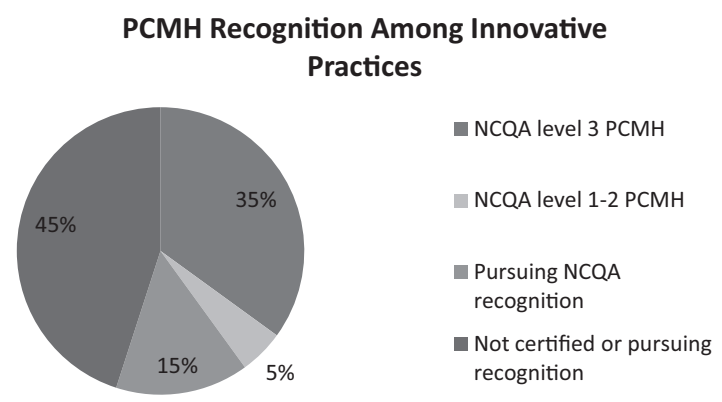

NCQA-recognized PCMHs would change the recognition process from a "check-the-box" formality to a genuine practice transformation.

\section{References}

1. National Committee of Quality Assurance. NCQA's patient-centered medical home (PCMH) 2011. January 31,2001. Available from: https:/www.ncqa.org/Portals/ 0/Programs/Recognition/PCMH_2011_Overview_5.2. pdf. Accessed April 6, 2012.

2. Dohan D, McCuistion MH, Frosch DL, Hung DY, Tai-Seale M. Recognition as a patient-centered medical home: fundamental or incidental? Ann Fam Med 2013;11(Suppl 1):S14-8.

3. Scholle SH, Asche SE, Morton S, Solberg LI, Tirodkar MA, Jaén CR. Support and strategies for change among small patient-centered medical home practices. Ann Fam Med 2013;11(Suppl 1):S6-13.

4. Stange KC, Nutting PA, Miller WL, et al. Defining and measuring the patient-centered medical home. J Gen Intern Med 2010;25:601-12.

5. Robert Wood Johnson Foundation. Thirty primary care practices selected as exemplary models of workforce efficiency and innovation. April 22, 2013. Available from: http://www.rwjf.org/en/about-rwjf/ newsroom/newsroom-content/2013/04/thirty-primarycare-practices-selected-as-exemplary-models-of-wo.html. Accessed March 5, 2014.

6. National Committee of Quality Assurance. First federally qualified health centers earn national recognition for patient-centered care for NCQA's new initiative. April 28, 2011. Available from: http://www.ncqa.org/Newsroom/ 2011NewsArchives/NewsReleaseApril282011.aspx. Accessed March 5, 2014. 